\title{
Síntesis de películas transparentes y conductoras utilizadas en celdas solares sensibilizadas por colorantes
}

\author{
Cesar Augusto Quiñones Segura* \\ Facultad de Ingeniería y Ciencias Básicas, Politécnico Grancolombiano. Bogotá, \\ Colombia
}

Fecha de entrega: Abril 12 De 2012

FECHa DE EVAluación: ABRil 30 DE 2012

FECHA DE APROBACIÓN: MAYO 3 DE 2012

\begin{abstract}
Resumen Este trabajo presenta la síntesis y caracterización de materiales transparentes y conductores utilizados en celdas solares fotoelectrocatalíticas. Específicamente, recubrimientos de óxido de Estaño conductor sintetizados por el método spray pyrolysis utilizando como precursores soluciones de cloruro de estaño y cloruro de Indio comerciales o fabricadas en el laboratorio. Las muestras se caracterizaron morfológicamente con microscopía de fuerza atómica, estructuralmente por difracción de rayos $\mathrm{x}$ y ópticamente con medidas de transmitancia espectral.
\end{abstract}

Palabras Clave: celdas solares sensibilizadas por colorante, DSSC, TCO, platino, spray pyrolysis.

\begin{abstract}
This paper presents the summary and characterization of transparent and conductor materials used in photoelectrocatalytic solar cells; more specifically, conducting tin oxide synthetized with the spray pyrolysis method, using commercial or lab-made tin chloride and indium chloride as precursors. The samples were morphologically characterized with atomic force microscopy, structurally by X-ray diffraction, and optically, with spectral transmittance measures.
\end{abstract}

Keywords: solar cells sensitized with coloring, DSSC, TCO, platinum, spray pyrolysis.

* Químico Universidad Nacional de Colombia. Doctor en Ciencias, Universidad Nacional de Colombia. Áreas de interés: electroquímica, celdas solares fotoelectroquímicas, fotocatálisis e instrumentación virtual. caquinones@poli.edu.co 


\section{Introducción}

En Octubre de 1991, Brian O'Regan y Michael Grätzel publicaron en la revista Nature un artículo reportando por primera vez resultados obtenidos con una novedosa celda solar basada en fotoelectrodo de óxido de titanio $\left(\mathrm{TiO}_{2}\right)$ sensibilizado por colorante que en adelante se conocería como celda solar de Gratzel o simplemente celda solar DSSC (dye sensitized solar cell) [1]; este tipo de celda solar fue especialmente atractiva desde su aparición debido a su eficiencia del 7,1-7,9\%. Este tipo de celda presentaba dos características novedosas: la primera, el óxido de titanio puede formar capas porosas de algunos micrómetros de espesor conformadas por nanoparticulas de 10-15 nm de tamaño, lo que permite lograr áreas superficiales de aproximadamente $780 \mathrm{~cm}^{2}$ por cada $\mathrm{cm}^{2}$ de área geométrica del foto electrodo. Por otra parte, la naturaleza del colorante utilizado, un complejo organometálico de rutenio, permitía una buena absorción del espectro de luz visible y una buena transferencia electrónica desde el colorante a las nanoparticulas de óxido de titanio. Así, se superaban dos de los principales inconvenientes de la generación fotovoltaica con este tipo de celdas y se inició un campo de investigación muy próspero que permitió en un tiempo muy corto lograr eficiencias superiores al $10 \%$ [2].

\subsection{Estructura de las celdas DSSC}

El la figura 1 se muestra un esquema de la estructura básica de la celda DSSC tipo Gratzel. Estas celdas están compuestas por tres elementos principales: Fotoelectrodo, contralectrodo y electrolito.

El fotoelectrodo es el elemento fundamental de la celda solar, el cual está conformado por un substrato de vidrio recubierto con una capa delgada de un óxido transparente y conductor (TCO), sobre el cual se deposita una capa delgada porosa de óxido de titanio nanoparticulado recubierto a su vez con un colorante. La función general del fotoelectrodo es generar pares electrón-hueco mediante absorción de la luz y facilitar el transporte de electrones hacia el contacto eléctrico a través de la capa de TCO; el contacto eléctrico debe ser óhmico para facilitar el tránsito de electrones desde el semiconductor hacia el circuito externo.

El contraelectrodo está conformado por un substrato de vidrio recubierto con una capa delgada de TCO, sobre el cual se deposita una capa delgada de platino. La función del contraelectrodo es cerrar el circuito eléctrico exterior de la celda para lo cual debe facilitar la transferencia electrónica desde la película de platino hacia el electrolito, con lo cual se induce una reacción de oxidación que regenera el electrolito.

El electrolito preferido en las celdas DSSC es una solución ionica del par redox yodo - triyoduro disuelto en un solvente orgánico, generalmente acetonitrilo. La función del electrolito es neutralizar las moléculas ionizadas del colorante, el cual, a su vez es regenerado por los electrones provenientes del circuito exterior que ingresan al electrolito a través del contraelectrodo de Pt. Aunque se ha realizado numerosos estudios para encontrar electrolitos poliméricos, sólidos o en gel, solamente los electrolitos líquidos han reportado altas eficiencias. 


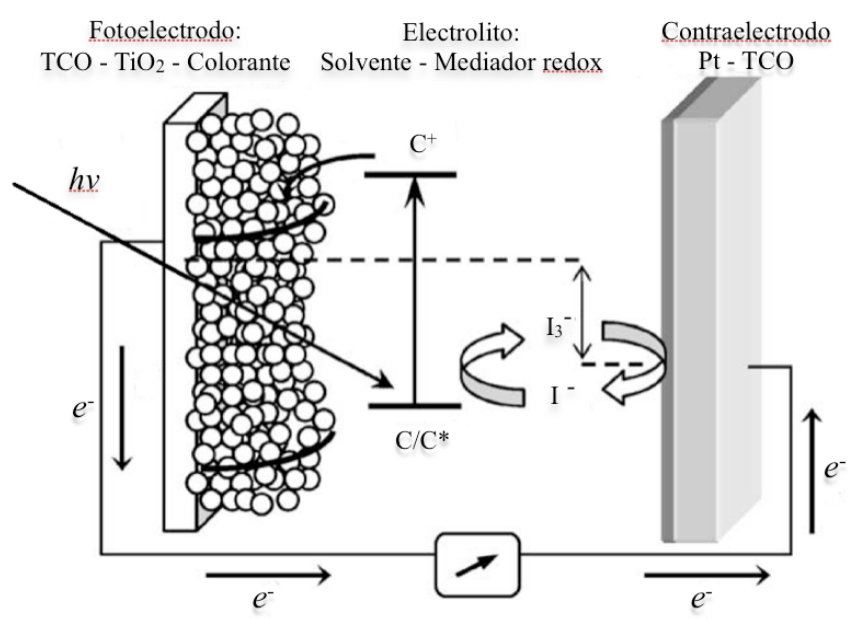

Figura 1. Estructura típica de una celda solar DSSC tipo Gratzel.

\subsection{Funcionamiento de las celdas solares DSSC}

La figura 2 muestra el diagrama de bandas de energía de una celda DSSC que incluye las bandas de energía del TCO y del óxido de titanio así como también los niveles asociados a los orbitales moleculares ocupado y desocupados del colorante y los correspondientes al par redox.

Se observan las bandas de valencia y de conducción del óxido de titanio características en materiales semiconductores, las cuales están separadas por la brecha de energía prohibida (band gap) del material, que en este caso es $3.2 \mathrm{eV}$. Para el colorante, la distribución energética de orbitales moleculares originados por la combinación de orbitales atómicos en la formación del enlace, genera una separación entre los orbitales moleculares más altos ocupados HOMO ( Hi Molecular Molecular Orbital) y los orbitales moleculares más bajos desocupados LUMO (Low Unnocupied Molecular Orbital). Para el par redox se visualiza el potencial intrínseco y para el proceso de óxido reducción llamado potencial estándar de reacción cuando se mide respecto al electrodo estándar de hidrógeno. Este potencial depende de la temperatura y de la concentraciones de las especies en su forma oxidada y reducida. Finalmente, el proceso de transferencia electrónica desde el contraelectrodo a la especie oxidada del par redox en solución, tienen un potencial característico que depende del material del cual está fabricado el contraelectrodo y de las propiedades de la superficie. Solamente cuando se tienen las combinaciones correctas de bandas de energía de cada uno de los componentes, se logra un apropiado funcionamiento del dispositivo.

El funcionamiento básico del dispositivo DSSC incluye los siguientes procesos: 


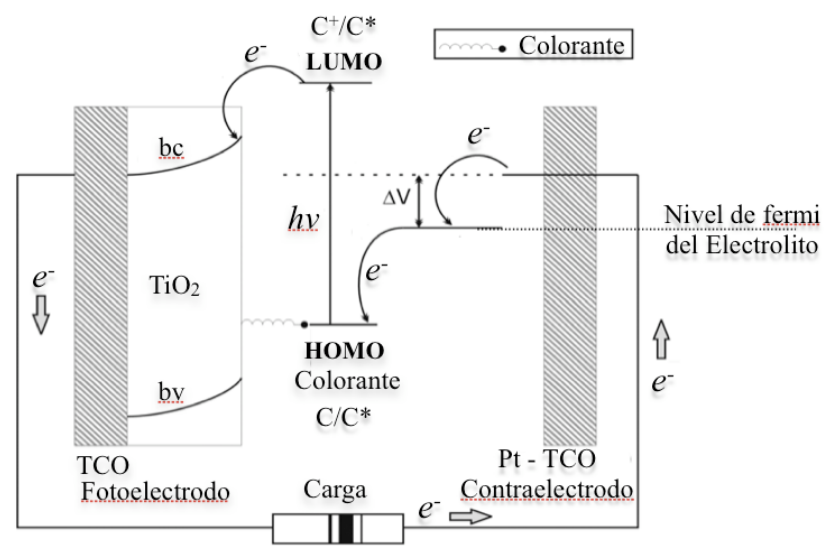

Figura 2. Diagrama de bandas de energía de una celda solar sensibilizada por colorante.

Absorción de un fotón por parte del colorante seguido de la generación de un excitón, cuando un electrón es excitado a un nivel más alto de energía de la estructura del colorante (excitación del electrón desde el orbital HOMO hasta el orbital LUMO de la molécula de colorante el cual es generalmente un orbital $\pi$ antienlazante).

Inyección del electrón desde el orbital LUMO a la banda de conducción del $\mathrm{TiO}_{2}$, generando un portador de carga libre y una molécula de colorante oxidada $\left(\mathrm{S}^{+} \longrightarrow \mathrm{S}^{*}+\mathrm{e}^{-}\right)$.

El electrón es transportado por difusión desde el $\mathrm{TiO}_{2}$ hasta alcanzar el TCO desde donde es inyectado al circuito externo para posteriormente ingresar al electrolito a través del contraelectrodo. La molécula oxidada de colorante es reducida por el mediador redox del electrolito, regresando así a su estado inicial que le permite ser nuevamente excitada por un fotón $\left(\mathrm{S}^{+}+\mathrm{e}^{-} \longrightarrow \mathrm{S}\right)$.

El mediador redox luego de reducir la molécula de colorante ha quedado oxidado y debe viajar hasta el contra electrodo para regenerarse (retomar su forma original) mediante la captura de un electrón por medio de una reacción electroquímica heterogénea. Este último paso completa el funcionamiento de la celda solar DSSC.

La separación entre el nivel HOMO y LUMO del colorante debe ser menor al band gap del óxido de titanio para que cumpla con la función de absorber una mayor parte del espectro visible. Además, el LUMO debe tener mayor nivel energético que la banda de conducción del semiconductor para que la transferencia electrónica pueda ocurrir. El nivel energético del par redox debe ser mayor que el nivel HOMO del colorante para que pueda ser regenerado. Finalmente, el potencial de óxido-reducción del par redox debe ser menor que el potencial 
ocurrido por la reacción de transferencia electrónica desde el contraelectrodo a la solución.

\subsection{Materiales conductores en las celdas DSSC}

Como se evidencia en la sección anterior, la celda solar fotoelectroquímica está compuesta por sustratos inertes y transparentes, materiales conductores, semiconductores, un sensibilizante y a diferencia de los demás tipos de celdas solares, un electrolito líquido. En el funcionamiento del dispositivo es importante la función de cada uno de ellos y en este artículo se tratará específicamente lo referente a los materiales conductores.

En el caso del fotoelectrodo es requisito que sea transparente y conductor, lo primero pues los fotones deben atravesar el fotoelectrodo para llegar al semiconductor, por tal motivo la transparencia es un factor de calidad importante en la síntesis de materiales utilizados para este tipo de películas. El otro parámetro de calidad importante es la resistencia eléctrica, siempre se busca que sea la más baja posible para que los electrones puedan circular fácilmente hacia el circuito eléctrico externo una vez que son recibidos por el $\mathrm{TiO}_{2}$. Así, en el fotoelectrodo se busca alta transparencia y baja resistencia eléctrica.

\section{Síntesis del óxido de indio dopado con indio (ITO)}

El óxido de Indio dopado con Estaño más conocido como ITO (Indium Tin Oxide) es un material semiconductor tipo n con una brecha de energía prohibida $\left(E_{g}\right)$ del orden de $3.7 \mathrm{eV}$, perteneciente al grupo de los materiales denominados TCO (óxidos transparentes-conductores) [3,4]. Se sintetizaron películas delgadas de ITO con propiedades adecuadas para usarlo como contacto eléctrico transparente en celdas solares DSSC. Las características más importantes del ITO son su baja resistividad (del orden de $10^{-4} \Omega \mathrm{cm}$ ) y la alta transparencia (del orden del $85 \%$ ).

Las películas delgadas de ITO pueden ser sintetizadas por diferentes técnicas entre las que se destacan: CVD [5], evaporación reactiva [6], Sputtering [7] y spray pyrolysis [8] entre otras. La técnica de spray pyrolysis una de las de mayor uso, por que se logran excelentes propiedades ópto-eléctricas y el costo de implementación de la técnica es muy bajo; ya que no se requiere vacío pues permite crecer capas delgadas mediante una reacción pirolítica de las especies contenidas en una solución finamente atomizada en un sustrato caliente $[9,10]$. La reacción química general para la formación de óxidos metálicos por el método de spray puede escribirse de la siguiente forma:

$$
\text { (MA)solución }+\mathrm{H}_{2} \mathrm{O} \longrightarrow \mathrm{MO}+2 \mathrm{HA}
$$

Donde M representa al metal y A al anión. La síntesis de películas delgadas por el método de spray pyrolysis involucra tres procesos básicos: (a) formación del spray de una solución que contiene las especies precursoras, (b) aspersión del spray sobre el sustrato caliente y (c) formación de la película mediante una 


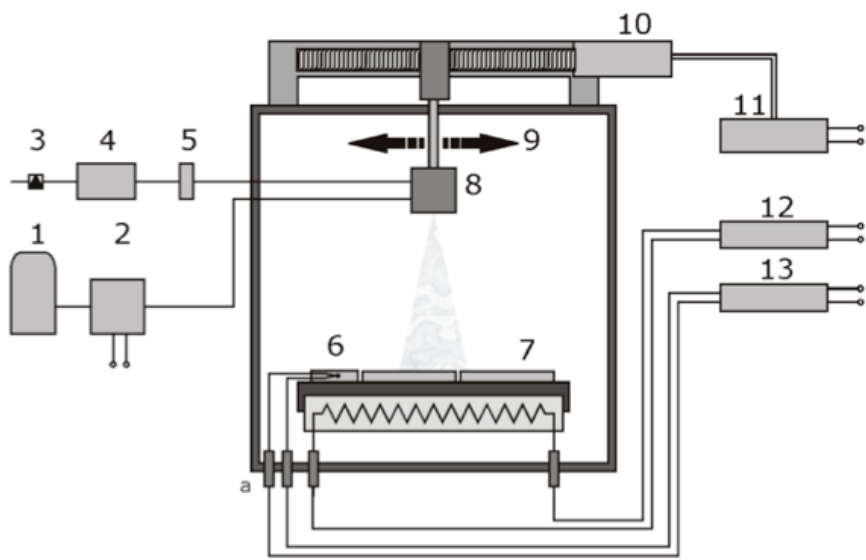

Figura 3. Diagrama del sistema de spray pyrolysis usado para la síntesis del ITO.

reacción pirolítica. En la figura 3 se muestra un diagrama del sistema usado en este trabajo para la síntesis del ITO por el método de spray pyrolysis.

Los componentes principales de este sistema son los siguientes: 1. Depósito de la solución que contiene las especies precursoras del ITO, 2. Bomba peristáltica usada para controlar el flujo de solución hacia la tobera, 3. Manómetro que permite regular la presión del gas de transporte, que en nuestro caso fue aire, 4. Filtros de aceite y agua para conseguir que a la tobera llegue aire seco libre de aceite, 5. Flujómetro para controlar el flujo de aire hacia la tobera, 6. Termocupla tipo K (incrustada en un testigo de vidrio) usada para medir la temperatura del sustrato a la cual ocurre la reacción pirolítica. Los sustratos empleados son de vidrio soda-lime de dimensiones $20 \mathrm{~cm} \times 40 \mathrm{~cm}$ 8. Tobera para producir el spray de la solución precursora del ITO, 11. Sistema de movimiento automático de la tobera que permite aumentar el área de deposición manteniendo una buena homogeneidad (en espesor y en propiedades) de las películas de ITO. Esto se realizó por medio de un sistema electrónico que permite a la tobera hacer un barrido lineal sobre el sustrato, el cual se repite cíclicamente en forma automática, mediante un motor y una guía $(9,10)$, 12. Sistema de calentamiento, consistente en una plancha de acero inoxidable que se calienta a través de una resistencia de cantal conectada a una fuente de potencia variable que suministra la corriente que permite controlar la temperatura del sustrato, 13. Display que indica el valor de la temperatura del sustrato.

\section{Influencia de parámetros de síntesis sobre las propiedades de películas delgadas de ITO}

En este trabajo se utilizaron como soluciones precursoras cloruros de indio y estaño de dos tipos. En el primer tipo las soluciones se prepararon a partir 
de los metales puros y acido clorhídrico. En el segundo caso, las sales para la preparación de las soluciones fueron adquiridas comercialmente como cloruros de alta pureza.

Las películas de ITO son usadas en celdas DSSC como material TCO por consiguiente es conveniente que este material sea altamente conductor y a la vez altamente transparente. Como bajo ciertas condiciones de síntesis, el aumento de conductividad eléctrica va acompañado de una disminución de la transmitancia, se usa una figura de mérito $\varphi_{M}$ definida en función de la transmitancia $T$ y de la resistividad eléctrica $\rho$ como método de diagnóstico para determinar los parámetros de síntesis que conducen a películas de ITO con propiedades adecuadas para ser usadas en celdas DSSC. Este parámetro es definido como $\varphi_{M}=T^{10} / \rho$ [11], el cual es adecuado para celdas solares debido a que ofrece más peso a la transmitancia.

Las curvas mostradas en la figura 4 muestran la influencia de los principales parámetros de síntesis (temperatura de sustrato $T s$, concentración molar de la especie precursora $\left[\operatorname{In}_{3}^{+}\right]$, concentración molar de la especie dopante $\left[\operatorname{Sn}_{2}^{+}\right] /\left[\operatorname{In}_{3}^{+}\right]$) sobre la transmitancia $T$ (medida en $\lambda=550 \mathrm{~nm}$ ), resistividad eléctrica $\rho$ y figura de mérito de las películas delgadas de ITO preparadas que usan tanto solución tipo I como solución tipo II.

Los resultados de la figura 4 muestran que todos los parámetros de síntesis estudiados afectan significativamente la transmitancia y la resistividad de las películas de ITO. Usando los datos de la figura de mérito, fue posible encontrar un conjunto de parámetros que permiten preparar películas de ITO con propiedades adecuadas para la aplicación deseada y se muestran en el cuadro 1.

\begin{tabular}{|c|c|c|c|c|c|c|c|}
\hline \multirow{2}{*}{ Material } & \multirow{2}{*}{ Solución } & \multirow{2}{*}[\operatorname{In}_{3}^{+}]{} & \multirow{2}{*}[\mathrm{Sn}_{2}^{+}]{} & \multirow{2}{*}{ Ts $\left({ }^{\circ} \mathrm{C}\right)$} & \multicolumn{3}{|c|}{ Resultados } \\
\hline & & & & & $T(\%)$ & $\rho(\Omega-\mathrm{cm})$ & $\Phi \mathrm{M}$ \\
\hline \multirow{2}{*}{ ITO } & $\begin{array}{l}\text { TIPO I. Preparada a } \\
\text { partir de } \mathrm{InCl}_{3} \text { y } \mathrm{SnCl}_{2} \\
\text { obtenidos por disolución } \\
\text { de metales en } \mathrm{HCl} \text {. }\end{array}$ & $0,4 \mathrm{M}$ & $0,016 \mathrm{M}$ & 440 & 90 & $6,7 \times 10^{-4}$ & 504 \\
\hline & $\begin{array}{l}\text { TIPO II. Preparada a } \\
\text { partir de } \mathrm{InCl}_{3} \text { y } \mathrm{SnCl}_{2} \\
\text { comerciales. }\end{array}$ & $0,5 \mathrm{M}$ & $0,025 \mathrm{M}$ & 450 & 86 & $6,0 \times 10^{-4}$ & 352 \\
\hline
\end{tabular}

Cuadro 1. Valores de los parámetros que condujeron a los mejores resultados para la síntesis de películas delgadas de ITO, usando los dos tipos de soluciones investigadas.

Los resultados del cuadro 1 muestran que con los dos tipos de soluciones se pueden sintetizar películas delgadas de ITO altamente transparentes y conductoras que son adecuadas para usarlas como contacto eléctrico transparente en celdas solares. Sin embargo las películas delgadas de ITO sintetizadas, que usan 

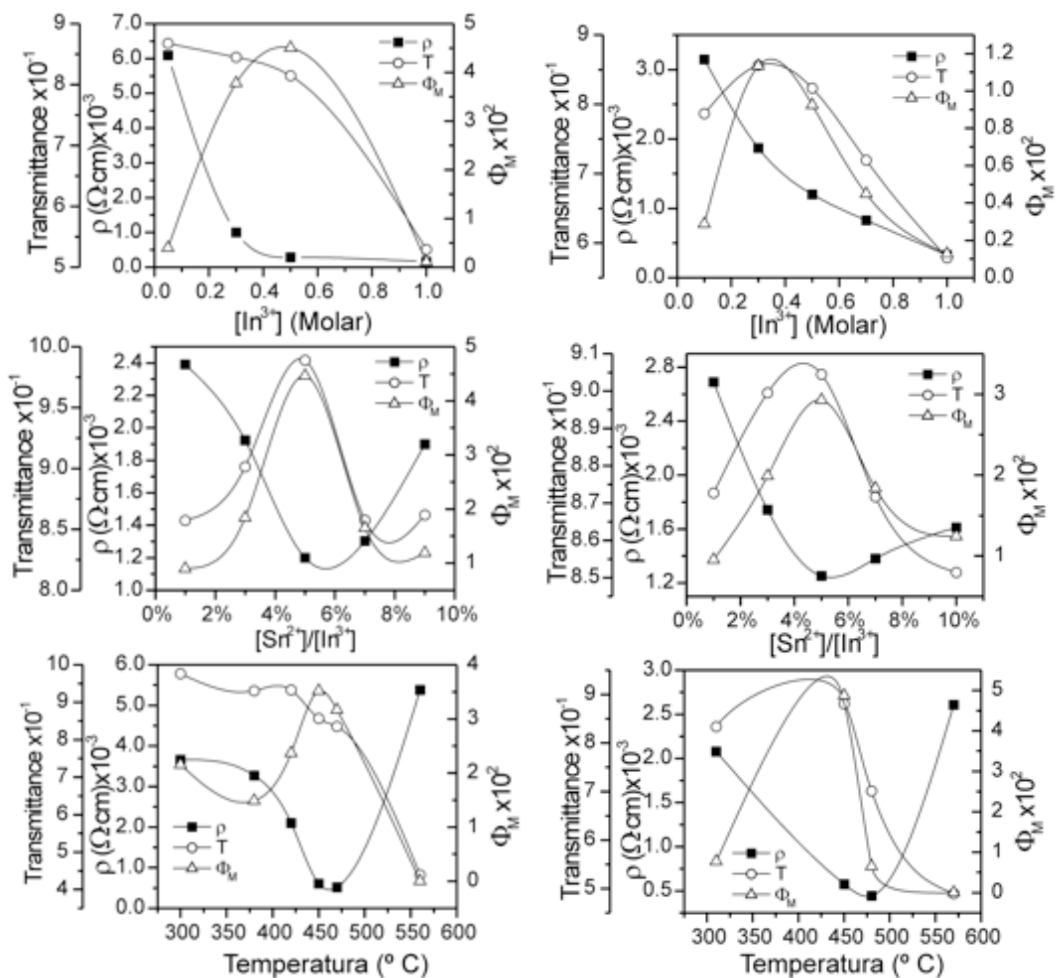

(a)

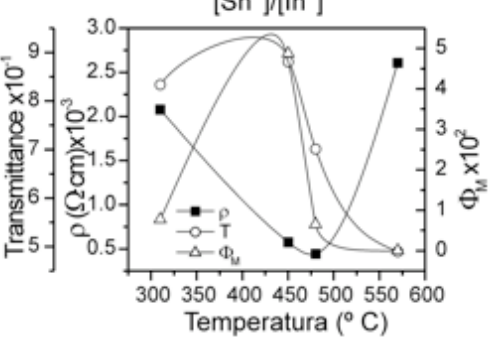

(b)

Figura 4. Influencia de los parámetros de síntesis $\left(T s,\left[\operatorname{In}_{3}^{+}\right]\right.$y $\left.\left[\mathrm{Sn}_{2}^{+}\right] /\left[\mathrm{In}_{3}^{+}\right]\right)$sobre la transmitancia, resistividad eléctrica y figura de mérito de películas delgadas de ITO sintetizadas, usando soluciones (a) Tipo I y (b) Tipo II.

la solución tipo I, presentan una figura de mérito mayor que las sintetizadas a partir de una solución preparada a partir de sales de In y Sn, grado analítico distribuidas comercialmente y que en el país son de difícil consecución y su costo es muy alto. Por el contrario, la síntesis del ITO a partir de una solución constituida por sales de In y de Sn, obtenidas por disolución de los metales en $\mathrm{HCl}$, se puede lograr fácilmente y a un costo muy bajo, ya que ésta se puede realizar con facilidad en el laboratorio.

En la figura 5 se muestra una curva de transmitancia espectral típica de las películas delgadas de ITO sintetizadas en este trabajo, usando la solución tipo I y los parámetros listados en el cuadro 1. Los resultados indicaron que en general, las películas delgadas de ITO con transmitancias mayores del $80 \%$ (en el visible y en el IR cercano) pueden ser obtenidas usando cualquiera de los dos tipos de soluciones estudiadas, indicando que las películas de ITO sintetizadas son adecuadas para su uso como contacto transparente y conductor de celdas DSSC. 


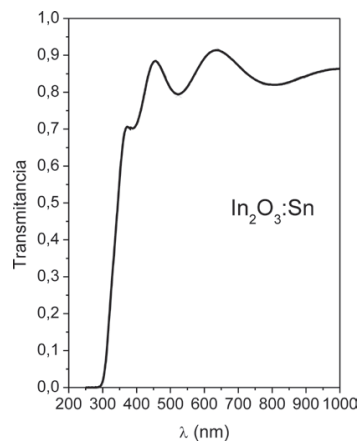

Figura 5. Transmitancia espectral típica de les películas delgadas de ITO sintetizadas dentro de este trabajo.

Las películas delgadas de ITO fueron caracterizadas adicionalmente a través de medidas de difracción de rayos-X (XRD) para determinar en qué fase y estructura cristalina crece este compuesto, así como también las correspondientes constantes de red. En la figura 6 se muestra un difractograma típico de las películas delgadas de ITO sintetizadas por spray pyrolysis, usando los parámetros listados en el cuadro 1.

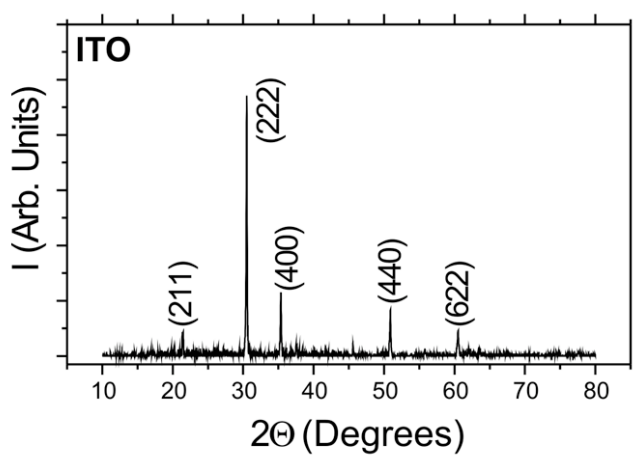

Figura 6. Espectro de difracción de rayos-x típico de películas delgadas de ITO altamente transparentes y conductoras, sintetizadas por spray pyrolysis.

Los resultados indican que independientemente del tipo de solución utilizada, las películas sintetizadas presentan únicamente reflexiones correspondientes a la fase $\mathrm{In}_{2} \mathrm{O}_{3}$ :Sn (ITO), las cuales crecen con estructura cúbica. En el cuadro 2 se presentan los resultados obtenidos de las medidas de XRD. 


\begin{tabular}{clcl}
\hline Muestra & Fase identificada & Estructura & $\begin{array}{l}\text { Constante } \\
\text { de red }(\AA)\end{array}$ \\
\hline ITO & $\mathrm{In}_{2} \mathrm{O}_{3}: \mathrm{Sn}(\mathrm{PDF}-\mathrm{Card}: 01-071-2195[59])$ & Cúbica & 10,117 \\
\hline
\end{tabular}

Cuadro 2. Fase, estructura cristalina y constantes de red correspondientes a películas delgadas de ITO preparadas por spray pyrolysis usando los parámetros listados en el cuadro 1.

Estudios realizados con microscopía AFM indicaron que en general la morfología de las muestras de ITO es afectada por los diferentes parámetros de síntesis, sin embargo el parámetro que más fuertemente afecta la morfología es la temperatura de síntesis. En la figura 7 se comparan las imágenes AFM correspondientes a películas delgadas de ITO sintetizadas sobre sustrato de vidrio usando la solución tipo I, bajo condiciones similares pero a temperatura de síntesis diferentes. Estos resultados muestran que la rugosidad y el tamaño de grano de las películas de ITO aumentan significativamente al aumentar la temperatura de síntesis. Las curvas graficadas en la figura 8 muestran cómo varía el valor promedio del tamaño de grano y de la rugosidad de las películas delgadas de ITO en dependencia de la temperatura de síntesis. Estos datos fueron obtenidos evaluando las imágenes AFM de la figura 7 con ayuda del programa ProScan Data.

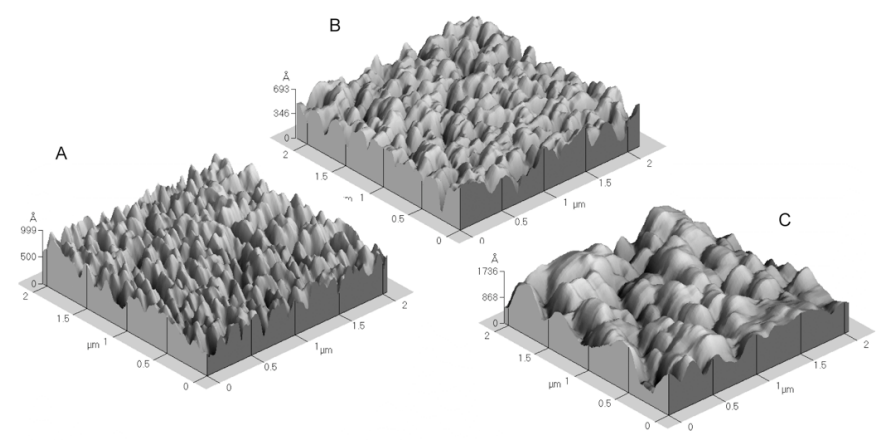

Figura 7. Imágenes AFM de películas delgadas de ITO depositadas a diferentes temperaturas: A) $\left.\left.350{ }^{\circ} \mathrm{C}, \mathrm{B}\right) 450{ }^{\circ} \mathrm{C}, \mathrm{C}\right) 500{ }^{\circ} \mathrm{C}$.

Los resultados de la figura 8 muestran que la temperatura de síntesis afecta significativamente la morfología de las películas de ITO; en particular, la rugosidad aumenta de 70 a $300 \mathrm{~nm}$ y el tamaño de grano 0,1 a 0,4 $\mu \mathrm{m}$ al aumentar la temperatura de síntesis de 350 a $500{ }^{\circ} \mathrm{C}$. 


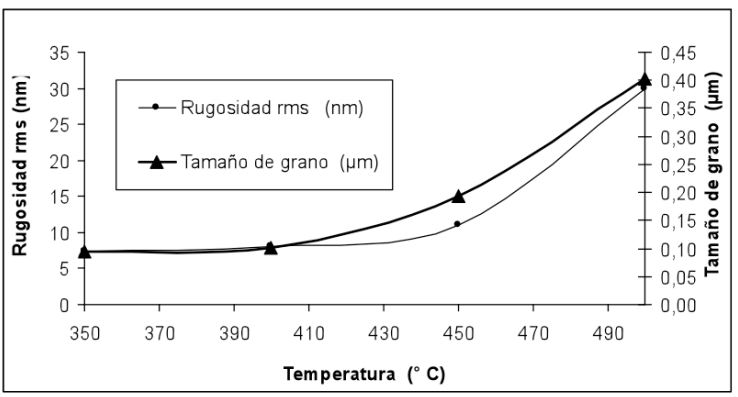

Figura 8. Variación del valor promedio del tamaño de grano y de la rugosidad (valor rms) de películas delgadas de ITO sintetizadas usando la solución tipo I, en dependencia de la temperatura de síntesis.

Correlacionando los resultados de la figura 8 con los de la figura 4 se puede deducir que la disminución de la resistividad y de la transmitancia del ITO, al aumentar la temperatura de síntesis, es en parte debido a un aumento de la rugosidad y del tamaño de grano. El aumento de rugosidad causa un aumento de la absorción de luz, lo que a su vez conduce a una disminución de la transmitancia; mientras que el incremento del tamaño de grano, causa una disminución de la densidad de fronteras de grano lo cual da lugar a un aumento de la movilidad de los portadores y con ello de la conductividad eléctrica. El aumento de la resistividad a temperaturas mayores de $450{ }^{\circ} \mathrm{C}$, parece ser causado por una reducción de vacancias de oxígeno que causan una compensación de las impurezas donoras producidas extrínsicamente mediante sustitución de In por Sn en la red del ITO.

\section{Conclusión}

Se sintetizaron películas transparentes y conductoras de óxido de Estaño dopado con Indio por la técnica de spray pyrolisis y soluciones preparadas a partir de sales comerciales y sales preparadas en el laboratorio. Las características de las películas preparadas con ambos tipos de soluciones cumplen con los requisitos necesarios para ser utilizados en celdas tipo DSSC. Sin embargo, las sales preparadas en el laboratorio produjeron mejores resultados.

\section{Referencias}

1. O'Regan, B., Grätzel, M.: Nature. 353, 737-739. (1991)

2. Bach, U., Lupo, D., Comte, P., Moser, J.E., Weissörtels, F., Salbeck, J., Spreitzer, H., Grätzel. M.: Nature. 395 583-585. (1998)

3. Chopra, K.L.; Thin Film Solar Cells, Prenium Press, New York and London, 195-270. 
4. Demichelis, F., Minetti-Mezzetti, E., Smurro, V., Tagliaferro A., Tresso, E.: Physical properties of chemically sprayed tin oxide and indium tin oxide transparent conductive films, J. Appl. Phys 18 1825-1832. (1985)

5. Chopra, K.L., Major, S., Pandya, D.K.: Transparent conductors - a status review, Thin Solid Films 102 1-46. (1983)

6. Habermeier, H.: Properties of Indium Tin Oxide thin films prepared by Reactive Evaporation, Thin Solid Films 80 157-160. (1981)

7. Kulkarni, A.K., Schulz, K.H., Lim, T.S., Khan, M.: Dependence of Sheet Resistance of Indium-Tin-Oxide Thin Films on Grain Size and Orientation Determined from X-ray Diffraction, Thin Solid Films 345 273-277. (1999)

8. Benamar, E., Rami, M., Messaoudi, C., Sayah, D., Ennaoui, A.: Structural, optical and electrical properties of indium tin oxide thin films prepared by spray pyrolysis, Solar Energy Materials and Solar Cells 56 125-139. (1999)

9. Puetz, J., Gasparro, G., Aegerter, M.A.: Liquid film spray deposition of transparent conducting oxide coatings, Thin Solid Films 442 40-43. (2003)

10. Rozati, S.M., Ganj, T.: Transparent conductive Sn-doped indium oxide thin films deposited by spray pyrolysis technique, Renewable Energy 29 1671-1676 (2004)

11. Haacke, G. : J. Appl. Phys. 474086 (1976) 